\title{
WEAKLY MAXIMAL DECIDABLE STRUCTURES
}

\author{
Alexis Bès ${ }^{1}$ AND PATriCK CÉGIElski ${ }^{1}$
}

\begin{abstract}
We prove that there exists a structure $M$ whose monadic second order theory is decidable, and such that the first-order theory of every expansion of $M$ by a constant is undecidable.
\end{abstract}

Mathematics Subject Classification. 03B25, 03C57, $03 \mathrm{D} 05$.

\section{INTRODUCTION}

In [3], Elgot and Rabin ask whether there exist maximal decidable structures, i.e. structures $M$ whose first-order theory is decidable and such that the first-order theory of any expansion of $M$ by a non-definable predicate is undecidable.

As far as we know, the only related results were obtained by Soprunov in [8], where he proves (by a forcing argument) that every structure in which a regular ordering is interpretable is not maximal. A partial ordering $(B,<)$ is said to be regular if for every $a \in B$ there exist distinct elements $b_{1}, b_{2} \in B$ such that $b_{1}<a$, $b_{2}<a$, and no element $c \in B$ satisfies both $c<b_{1}$ and $c<b_{2}$. As a corollary he also proves that there is no maximal decidable structure if we replace "first-order theory" by "weak monadic second-order theory".

In this paper we consider a weakening of Elgot-Rabin's question, namely the question whether there exist maximal decidable theories with respect to nondefinable constants. We answer positively this question for first-order theories as well as monadic second-order theories, by proving that there exists a structure $M$ with a decidable monadic second-order theory, and such that every expansion of $M$ by a constant has an undecidable first-order theory. The proof makes use of Shelah's composition theorem and Büchi's result on the monadic second order theory of $(\mathbb{N},<)$.

Keywords and phrases. Decidability, first-order theories, monadic second-order theories, maximality, automata, rich words.

${ }^{1}$ LACL, EA 4213, Université Paris-Est, Faculté des Sciences et Technologie, 61 avenue du Général de Gaulle, 94010 Créteil Cedex, France; [bes, cegielski] @univ-paris12.fr

(c) EDP Sciences 2007 
We also prove that $M$ is not maximal in the sense of Elgot and Rabin, even if we replace "first-order theory" by "monadic second-order theory".

\section{Some useful tools}

Given a structure $M$ we denote by $F O(M)$ (respectively $M S O(M)$ ) the firstorder (respectively monadic second-order) theory of $M$. The base set of $M$ is denoted by $|M|$. We shall sometimes confuse logical symbols and their interpretation.

Given a relational language $L$ and a countable $L$-structure $M$, we say that $M$ is computable if its atomic diagram is computable, i.e. if there exists an injective map $f:|M| \rightarrow \mathbb{N}$ such that the range of $f$, as well as all sets $\left\{\left(f\left(a_{1}\right), \ldots, f\left(a_{n}\right)\right)\right.$ : $\left.M \models R\left(a_{1}, \ldots, a_{n}\right)\right\}$ for every relation $R$ of $L$, are recursive (see e.g. [6]).

\subsection{Extensions of THE MONADiC SECOND-ORDER THEORY OF $(\mathbb{N},<)$}

The following proposition is an easy corollary of Büchi's result [1] about the monadic second order theory of $(\mathbb{N},<)$.

Proposition 1.1. Let $R$ be a unary predicate, and let $<$ be interpreted as the natural ordering over natural numbers. It is decidable whether a MSO sentence $\phi$ over the language $\{<, R\}$ admits a model of the form $(\mathbb{N} ;<, R)$.

Moreover if it admits such a model then it admits a model where the interpretation of $R$ is an ultimately periodic subset of $\mathbb{N}$ (which can be computed effectively from $\phi)$.

Proof. By [1] for every monadic second-order sentence $\phi$ there exists an automaton $\mathcal{A}$ over $\omega$-words such that $(\mathbb{N},<, R) \models \phi$ if and only if the word $w_{R} \in\{0,1\}^{\omega}$ which corresponds to the characteristic sequence of $R$ is recognized by $\mathcal{A}$. Moreover $\mathcal{A}$ can be computed effectively from $\phi$. Thus the question whether a MSO sentence $\phi$ over the language $\{<, R\}$ admits a model of the form $(\mathbb{N} ;<, R)$ is reducible to the emptiness problem for $\mathcal{A}$, which is decidable by [1].

The second part of the proposition comes from the fact that if the automaton $\mathcal{A}$ accepts some $\omega$-word then it accepts some ultimately periodic $\omega$-word, which can be computed effectively from $\mathcal{A}$.

We shall use the notion of rich word (see $[2,5])$.

Definition 1.2. Given a finite alphabet $A$, a $\mathbb{Z}$-word over $A$ is a sequence $w$ : $\mathbb{Z} \rightarrow A$. We say that a $\mathbb{Z}$-word $w$ is rich if every finite word over $A$ appears infinitely many times in $w$ in both directions; that is, for every non-empty word $u=u_{1} \ldots u_{n}$ over $A$ and every $j \in \mathbb{Z}$ there exists $k>j$ and $k^{\prime}<j$ such that $w(k+1) \ldots w(k+n)=u$ and $w\left(k^{\prime}+1\right) \ldots w\left(k^{\prime}+n\right)=u$.

Compton proved that the MSO theory of $(\mathbb{Z},<)$ extended by any rich word is decidable [2]: 
Theorem 1.3. Let $w: \mathbb{Z} \rightarrow A$ be a rich word. For every $a \in A$ let $X_{a} \subseteq \mathbb{Z}$ be defined as $X_{a}=\{z \in \mathbb{Z}: w(z)=a\}$. Then the $M S O$ theory of $\left(\mathbb{Z} ;<,\left(X_{a}\right)_{a \in A}\right)$ is decidable.

\subsection{The COMposition Method}

We shall use Shelah's composition method [7] which allows to reduce the MSO theory of a sum of structures to the MSO theories of the components and the MSO theory of the index structure. This method is a generalization of the FefermanVaught technique [4]. For a recent and detailed presentation of Shelah's method we refer the reader to [10], from which we borrow most of our definitions and notations.

We state definitions and results for the special case where the language $L$ of the component structures equals $L=\{<, R\}$ where $R$ denotes a unary predicate (as in [10]).

Definition 1.4. We start from an index structure $I n d=\left(I,<^{I}\right)$ where $<^{I}$ is a linear ordering. Consider the relational language $L=\{<, R\}$ where $R$ denotes a unary predicate, and a family $\left(M_{i}\right)_{i \in I}$ of $L$-structures $M_{i}=\left(A_{i} ;<^{i}, R^{i}\right)$ such that the interpretation $<^{i}$ of $<$ in each $M_{i}$ is a linear ordering. We define the ordered sum of the family $\left(M_{i}\right)_{i \in I}$ as the $L$-structure $M=\left(A ;<^{M}, R^{M}\right)$ where

- $A$ equals the disjoint union of the $A_{i}$ 's,

- $x<^{M} y$ holds if and only if $\left(x \in A_{i}\right.$ and $y \in A_{j}$ for some $\left.i<^{I} j\right)$, or $\left(x, y \in A_{i}\right.$ and $\left.x<^{i} y\right)$,

- for every $x \in A, R^{M}(x)$ holds if and only if $M_{i} \models R^{i}(x)$ where $i$ is such that $x \in A_{i}$.

We shall use the notation $M=\sum_{i \in I} M_{i}$.

In view of Theorem 1.7 one has to consider a slight modification of the MSO formalism:

- we deal with MSO logic in which only monadic second-order variables occur; this is always possible by introducing the predicate "is a singleton" and replacing each relation which involves FO variables by relations which involve MSO variables restricted to singletons;

- for every $n \geq 1$ we add to the language the predicates $\operatorname{Cov}_{n}\left(X_{1}, \ldots, X_{n}\right)$ and $\operatorname{Part}_{n}\left(X_{1}, \ldots, X_{n}\right)$, which are respectively interpreted as " $X_{1} \cup \cdots \cup$ $X_{n}$ equals the whole domain", and " $\left(X_{1}, \ldots, X_{n}\right)$ is a partition of the domain". These predicates do not add any expressive power to the full MSO theory since they are already MSO-definable, but their use allows to extend the expressive power of quantifier-free formulas.

Definition 1.5. Given $n \in \mathbb{N}$ and a sequence $\bar{k}=\left(k_{1}, \ldots, k_{n}\right)$ of natural numbers, we call $\bar{k}$-formula a MSO formula in prenex form which consists in a block of $k_{1}$ quantifiers of the same kind, followed by a block of $k_{2}$ quantifiers of the same kind, etc... and ends with a block of $k_{n}$ quantifiers of the same kind followed by 
a quantifier-free formula. For example the formula $\forall X_{1} \forall X_{2} \exists X_{3} \phi(\bar{X})$, where $\phi$ is quantifier-free, is a $(2,1)$-formula. A $\bar{k}$-sentence is a $\bar{k}$-formula which is closed.

Definition 1.6. The $\bar{k}$-theory of a structure $M$, which we denote by $T^{\bar{k}}(M)$, is the set of $\bar{k}$-sentences which are true in $M$. We denote $M \equiv_{\bar{k}} M^{\prime}$ if $M$ and $M^{\prime}$ have the same $\bar{k}$-theory.

Observe that the equivalence classes of $\equiv_{\bar{k}}$ can be defined in MSO logic: for every set $T$ of $\bar{k}$-sentences there exists a $\bar{k}$-sentence $\psi_{T}$ such that $M \models \psi_{T}$ if and only if the $\bar{k}$-theory of $M$ equals $T$. Moreover $\psi_{T}$ can be computed effectively from $T$.

We state Shelah's composition theorem [7] as in [10]:

Theorem 1.7. Let $M=\sum_{i \in I} M_{i}$ be the ordered sum of the family $\left(M_{i}\right)_{i \in I}$. Given a sequence $\bar{k}=\left(k_{1}, \ldots, k_{n}\right)$ one can compute a sequence $\bar{r}=\left(r_{1}, \ldots, r_{n}\right)$ such that the $\bar{k}$-theory of $M$ is determined (in an effective way) by the $\bar{r}$-theory of the structure $\left(I,<^{I}, Q_{1}, \ldots, Q_{p}\right)$ where

$$
Q_{j}=\left\{i \in I: T^{\bar{k}}\left(M_{i}\right)=\tau_{j}\right\} \quad j=1, \ldots, p
$$

and $\tau_{1}, \ldots, \tau_{p}$ denote the $p$ distinct $\bar{k}$-theories of the $M_{i}$ 's.

\section{A WeAKly maXimal DECidABle StRUCture}

We state now the main result of the paper.

Theorem 2.1. There exist a relational language $L$ and a countable L-structure $M$ such that:

- $M$ is computable;

- the monadic second-order theory of $M$ is decidable;

- every $(L \cup\{c\})$-expansion of $M$ by a constant symbol $c$ has an undecidable first-order theory.

Proof. Let us state first the main lines of the proof. We shall define $M$ as an ordered sum (indexed by $\mathbb{Z}$ with its natural ordering) of structures of the form $(\mathbb{N} ;<, R)$ where $R$ is some unary predicate whose interpretation varies from one component to another. The idea is to "hide" a copy of some structure $(\mathbb{N} ;<, B)$ among other components of the ordered sum, in such a way that, on one hand, this copy cannot be distinguished easily from other components by a MSO sentence, and on the other hand the structure $(\mathbb{N} ;<, B)$ has an undecidable FO theory. The undecidability of $F O(M)$ enriched by any constant will come from the fact that the constant allows to localize the component to which it belongs, from which we can reach the copy of $(\mathbb{N} ;<, B)$ and use the undecidability of $F O(\mathbb{N} ;<, B)^{1}$.

\footnotetext{
${ }^{1}$ The idea of using designated elements to get undecidability already appears in [9], Theorem 4.5.
} 
The decidability of $M S O(M)$ will be obtained by using Theorem 1.7, which will allow to reduce the decision problem for a given MSO sentence $\phi$ to the decision problem for an extension of $M S O(\mathbb{Z},<)$ by a rich word, which is decidable by Theorem 1.3.

Let $W \subseteq \mathbb{N}$ be a recursively enumerable not recursive predicate. Let $g_{W}: \mathbb{N} \rightarrow$ $\mathbb{N}$ be an enumerator for $W$, i.e. a total recursive function whose range is $W$. Let $B \subseteq \mathbb{N}$ be the set of elements of the sequence $\left(b_{n}\right)_{n \in \mathbb{N}}$ defined by $b_{0}=g_{W}(0)$ and $b_{n+1}=b_{n}+g_{W}(n)+1$.

Lemma 2.2. $F O(\mathbb{N} ;<, B)$ is undecidable.

Proof. One can associate effectively to every $m \in \mathbb{N}$ some FO-sentence $\phi_{m}$ such that $m \in W$ if and only if $(\mathbb{N} ;<, B) \models \phi_{m}$; this sentence simply expresses the fact that there exist two consecutive elements of $B$ at distance $m+1$.

Let us define the structure $M$. Consider a recursive function $f$ which enumerates all finite non-empty sequences of ultimately periodic subsets of $\mathbb{N}$ (such a function exists). Given $n \in \mathbb{N}$ we denote by $l(n)$ the length of the sequence $f(n)$.

Definition 2.3. Let $\left(R^{i}\right)_{i \in \mathbb{Z}}$ be the sequence of subsets of $\mathbb{N}$ defined by the following conditions:

- $R^{0}=B$

- $f(0)=\left(R^{1}, R^{2}, \ldots, R^{l(0)}\right)$;

- for every $n \geq 1$, if we set $s_{n}=\sum_{0 \leq j<n} l(2 j)$ then

$$
f(2 n)=\left(R^{s_{n}+1}, R^{s_{n}+2}, \ldots, R^{s_{n}+l(2 n)}\right) ;
$$

- $f(1)=\left(R^{-l(1)}, R^{2}, \ldots, R^{-1}\right)$;

- for every $n \geq 1$, if we set $s_{n}^{\prime}=\sum_{0 \leq j<n} l(2 j+1)$ then

$$
f(2 n+1)=\left(R^{-s_{n}^{\prime}-l(2 n+1)}, \ldots, R^{-s_{n}^{\prime}-1}\right) .
$$

The above definition is illustrated in Figure 1.

$\cdots \underbrace{R^{-l(1)-l(3)} \cdots R^{-l(1)-1}}_{f(3)} \underbrace{R^{-l(1)} \cdots R^{-1}}_{f(1)} R^{0} \underbrace{R^{1} \cdots R^{l(0)}}_{f(0)} \underbrace{R^{l(0)+1} \cdots R^{l(0)+l(2)}}_{f(2)} \cdots$

Figure 1. The sequence $\left(R^{i}\right)_{i \in \mathbb{Z}}$.

Definition 2.4. We define the $\{<, R\}$-structure $M=\sum_{i \in \mathbb{Z}} M_{i}$, where $M_{i}=$ $\left(\mathbb{N} ;<, R^{i}\right)$ for every $i \in \mathbb{Z}$.

Let us prove that $M$ satisfies the conditions stated in Theorem 2.1. The proof of the following lemma is a straightforward consequence of the two previous definitions and the fact that the set $B$ is recursive. 
Lemma 2.5. The structure $M$ is computable.

Let us prove now that $M$ is maximal with respect to constants.

Lemma 2.6. Let $c$ be a constant symbol. For any interpretation of $c$, the structure $M^{\prime}$ defined as the expansion of $M$ with respect to $c$ has an undecidable first-order theory.

Proof. Using the constant $c$ one can easily define in $M^{\prime}$ the first element $x$ of the $M_{i}$ to which $c$ belongs: this is the greatest element less than or equal to $c$ and without predecessor. Then from $x$ one can define the first element $y$ of $M_{0}$ (seen as an element of $|M|)$ : in case $i \geq 0$ then the formula expresses that $y \leq x, y$ has no predecessor, and there are $i$ elements without predecessor between $y$ and $x$; the case $i<0$ is similar. Then from $y$ one defines easily the subset $\left|M_{0}\right|$ (seen as a subset of $|M|)$. Finally since $M_{0}$ is a copy of $(\mathbb{N} ;<, B)$, classical relativization techniques allow to translate every $L$-sentence $\varphi$ in a $(L \cup\{c\})$-sentence $\varphi^{\prime}$ such that $(\mathbb{N} ;<, B) \models \varphi$ if and only if $M^{\prime} \models \varphi^{\prime}$. This proves that $F O(\mathbb{N} ;<, B)$ is interpretable into $F O\left(M^{\prime}\right)$, and Lemma 2.2 yields the result.

Note that the previous arguments can be extended in an easy way to the case where one expands $M$ by any unary predicate $R$ whose interpretation is a finite or co-finite subset of $|M|$.

Lemma 2.7. The MSO theory of $M$ is decidable.

Proof. Let us prove that the application which maps $\bar{k}$ to the $\bar{k}$-theory of $M$ is effective. By Theorem 1.7 it is sufficient to prove that for every $\bar{k}$

(1) one can compute effectively the set $S(\bar{k})=\left\{T^{\bar{k}}\left(M_{i}\right) \mid i \in \mathbb{Z}\right\}$;

(2) if we denote by $\tau_{1}, \ldots, \tau_{p_{\bar{k}}}$ the distinct elements of $S(\bar{k})$, then the MSO theory of the structure $\left(\mathbb{Z} ;<, Q_{1}, \ldots, Q_{p_{\bar{k}}}\right)$, where

$$
Q_{j}=\left\{i \in \mathbb{Z}: T^{\bar{k}}\left(M_{i}\right)=\tau_{j}\right\}
$$

is decidable.

First of all, observe that for every set $\tau$ of $\bar{k}$-sentences there exists a sentence $\phi$ such that for every $L$-structure $Z$, we have $Z=\phi$ if and only if $T^{\bar{k}}(Z)=\tau$. Now by Proposition 1.1 we can check effectively whether $\phi$ admits a model $Z$ of the form $(\mathbb{N} ;<, R)$ where $<$ is interpreted as the natural ordering over $\mathbb{N}$. If such a model $Z$ exists, then Proposition 1.1 implies that there exists an ultimately periodic set $X \subseteq \mathbb{N}$ such that $(\mathbb{N} ;<, R) \models \phi$ when one interprets $R$ by $X$, and thus by definition of the $M_{i}$ 's there exists $i \in \mathbb{Z}$ such that $M_{i}=\phi$, i.e. $T^{\bar{k}}\left(M_{i}\right)=\tau$. On the other hand if no such model $Z$ exists then a fortiori there is no $M_{i}$ such that $T^{\bar{k}}\left(M_{i}\right)=\tau$. This proves $(1)$.

Let us prove (2). By Theorem 1.3 it suffices to prove that the word $w: \mathbb{Z} \rightarrow$ $\left\{1, \ldots, p_{\bar{k}}\right\}$ defined by $w(z)=i$ if and only if $z \in Q_{i}$, is a rich word. This amounts to show that for all $n, j \in \mathbb{N}$ and every non-empty sequence $\left(t_{1}, \ldots, t_{n}\right)$ of elements of $\left\{\tau_{1}, \ldots, \tau_{p_{\bar{k}}}\right\}$, there exist $z>j$ (resp. $z<j$ ) such that the $\bar{k}$-theories of the 
structures $M_{z+1}, \ldots, M_{z+n}$ are $t_{1}, \ldots, t_{n}$, respectively. Now by Proposition 1.1 it suffices to prove that for every sequence $\left(F_{1}, \ldots, F_{n}\right)$ of ultimately periodic subsets of $\mathbb{N}$ there exist $z>j$ (resp. $z<j$ ) such that $R^{z+j}=F_{j}$ for every $j \in\{1, \ldots, n\}$. This is a direct consequence of the definition of the sequence $\left(R^{i}\right)_{i \in \mathbb{Z} \text {. }}$

This completes the proof of Theorem 2.1. This theorem shows that the structure $M$ is maximal with respect to constants. However $M$ is not maximal in the sense of Elgot and Rabin, even if we consider monadic second-order logic.

Proposition 2.8. There exists $S \subseteq|M|$ which is not $M S O$-definable in $M$, and such that the $(L \cup\{S\})$-expansion of $M$ induced by $S$ has a decidable MSO-theory.

Proof. The set $S$ is defined by induction on $n \in \mathbb{N}$. The idea is to mark for each $n$ finitely many components $M_{i}$ of $M$, either in $S$ or in the complement of $S$ (i.e. either all elements, or no element, of $M_{i}$ belong to $S$ ).

For every $i \in \mathbb{Z}$ we denote by $S^{i}$ the interpretation of $S$ in the structure $M_{i}$, and by $M_{i}^{\prime}$ the $(L \cup\{S\})$-expansion of $M_{i}$ induced by $S^{i}$.

Let $g$ be a function which enumerates all pairs $(H, B)$ where $H$ denotes a finite and non empty sequence of ultimately periodic subsets of $\mathbb{N}$, and $B$ is a subset of $\{1, \ldots, m\}$ where $m$ denotes the length of $H$ (such a function exists). We shall use the notation $g(n)=\left(\left(H_{n, 1}, H_{n, 2}, \ldots, H_{n, \lambda(n)}\right), B_{n}\right)$.

For every $n \in \mathbb{N}$ we proceed as follows:

(1) Consider the set $\mathcal{F}_{n}$ of MSO formulas $\varphi(X)$ in the language $L$ with one free variable $X$, with quantifier rank less than or equal to $n$, and such that the subsets $X \subseteq|M|$ for which $M \models \varphi(X)$ holds are unions of sets $\left|M_{i}\right|$. The language $L$ is finite and relational, thus up to equivalence there exist finitely many formulas in $\mathcal{F}_{n}$. Therefore there exist $a_{n} \geq 1$ and a partition $\left(Y_{n, 1}, \ldots, Y_{n, a_{n}}\right)$ of $|M|$ such that every $Y_{n, i}$ is definable by a formula of $\mathcal{F}_{n}$, and every set $X \subseteq|M|$ which is definable by a formula in $\mathcal{F}_{n}$ is a finite union of some of the $Y_{n, i}$ 's. Since $|M|$ is infinite there exists $j$ such that $Y_{n, j}$ is the union of infinitely many sets $\left|M_{i}\right|$. Let $j^{\prime}$ be the least positive integer such that $\left|M_{j^{\prime}}\right| \subseteq Y_{n, j}$ and $M_{j^{\prime}}$ is not marked yet. We mark all elements of $\left|M_{j^{\prime}}\right|$ in $S$, that is we set $S^{j^{\prime}}=\left|M_{j^{\prime}}\right|$. Now let $j^{\prime \prime}$ be the least positive integer greater than $j^{\prime}$ and such that $\left|M_{j^{\prime \prime}}\right| \subseteq Y_{n, j}$ and $M_{j^{\prime \prime}}$ is not marked yet. We mark all elements of $\left|M_{j^{\prime \prime}}\right|$ in the complement of $S$, that is we set $S^{j^{\prime \prime}}=\varnothing$.

This step of the marking ensures that both $S$ and its complement intersect $Y_{n, j}$, which implies (by definition of the partition $\left(Y_{n, 1}, \ldots, Y_{n, a_{n}}\right)$ ) that $S$ is not definable by any formula of $\mathcal{F}_{n}$.

(2) Let $k$ be the least positive integer such that no $M_{t}$ with $t \geq k$ is marked yet, and moreover $R^{k+i}=H_{n, i}$ for every $i \in\{1, \ldots, \lambda(n)\}$. By definition of $M$ such a $k$ exists. For every $i \in\{1, \ldots, \lambda(n)\}$ we set $S^{k+i}=\left|M^{k+i}\right|$ if $i \in B_{n}$, and $S^{k+i}=\varnothing$ otherwise. Now let $k^{\prime}$ be the greatest negative integer such that no $M_{t}$ with $t<k^{\prime}+\lambda(n)$ is marked yet, and moreover $R^{k^{\prime}+i}=H_{n, i}$ for every $i \in\{1, \ldots, \lambda(n)\}$. By definition of $M$ such a $k^{\prime}$ 
exists. For every $i \in\{1, \ldots, \lambda(n)\}$ we set $S^{k^{\prime}+i}=\left|M^{k^{\prime}+i}\right|$ if $i \in B_{n}$, and $S^{k^{\prime}+i}=\varnothing$ otherwise.

(3) For every $i \in\left[k^{\prime}, k\right]$ such that $M_{i}$ is not marked yet, we mark $M_{i}$ in the complement of $S$, i.e. we set $S^{i}=\varnothing$.

The proof that $S$ is not MSO-definable in $M$ easily follows from step (1) of the construction.

Let $M^{\prime}$ denote the $(L \cup\{S\})$-expansion of $M$ induced by $S$. The proof that $M S O\left(M^{\prime}\right)$ is decidable is similar to the one of Lemma 2.7. Let us state the main arguments. We have to prove the following:

- the function $h: \bar{k} \rightarrow\left\{T^{\bar{k}}\left(M_{i}^{\prime}\right) \mid i \in \mathbb{Z}\right\}$ is recursive;

- for all $\bar{k}, m \geq 1$, every sequence $\left(\tau_{1}, \ldots, \tau_{m}\right)$ of elements of $h(\bar{k})$, and every $j \in \mathbb{Z}$, there exist $z>j$ (resp. $z<j$ ) such that the $\bar{k}$-theories of the structures $M_{z+1}^{\prime}, \ldots, M_{z+m}^{\prime}$ are $\tau_{1}, \ldots, \tau_{m}$, respectively.

For every $i \in \mathbb{Z}$, let $a_{i}=1$ if $S^{i}=\left|M_{i}\right|$, and $a_{i}=0$ otherwise. It is easy to prove that for every $i$, the $\bar{k}$-theory of the $(L \cup\{S\})$-expansion $M_{i}^{\prime}$ of $M_{i}$ induced by $S$ can be computed effectively from $a_{i}$ and the $\bar{k}$-theory of $M_{i}$. This fact together with Proposition 1.1 yields that $h$ is recursive.

For the second item, by Proposition 1.1 it suffices to prove that for every $m \geq 1$, for every $m$-tuple $\left(E_{1}, \ldots, E_{m}\right)$ of ultimately periodic subsets of $\mathbb{N}$ and every $m$ tuple $\left(b_{1}, \ldots, b_{m}\right)$ of elements of $\{0,1\}$, there exist infinitely many positive integers and infinitely many negative integers $i$ such that for every $j \in\{1, \ldots, m\}$,

- $R^{i+j}=E_{j}$

- $S^{i+j}=\left|M_{i+j}\right|$ if $b_{j}=1$, and $S^{i+j}=\varnothing$ if $b_{j}=0$.

This fact is a direct consequence of the step (2) in the construction of $S$.

Combining the two items above with Theorem 1.3 and Theorem 1.7 (as in the proof of Lemma 2.7) yields the required result.

Let us note that in the proof above, we show the existence of $S$ but do not prove that one can define $S$ effectively, i.e. that the structure $M^{\prime}$ is computable. We do not know whether such an effective definition is possible.

Acknowledgements. We dedicate the paper to Serge Grigorieff on his 60th birthday. We wish to thank Andrei Paskevich who kindly translated Soprunov's paper, and the anonymous referee for useful suggestions. 


\section{REFERENCES}

[1] J.R. Büchi, On a decision method in the restricted second-order arithmetic. In Proc. Int. Congress Logic, Methodology and Philosophy of science, Berkeley 1960. Stanford University Press (1962) 1-11.

[2] K.J. Compton, On rich words. In M. Lothaire, editor, Combinatorics on words. Progress and perspectives, Proc. Int. Meet., Waterloo, Canada (1982). Encyclopedia of Mathematics 17, Addison-Wesley (1983) 39-61.

[3] C.C. Elgot and M.O. Rabin. Decidability and undecidability of extensions of second (first) order theory of (generalized) successor. J. Symbolic Logic 31 (1966) 169-181.

[4] S. Feferman and R.L. Vaught, The first order properties of products of algebraic systems. Fund. Math. 47 (1959) 57-103.

[5] D. Perrin and J.-É. Pin, Infinite Words. Pure Appl. Math. 141 (2004).

[6] V.S.Harizanov, Computably-theoretic complexity of countable structures. Bull. Symbolic Logic 8 (2002) 457-477.

[7] S. Shelah, The monadic theory of order. Ann. Math. 102 (1975) 379-419.

[8] S. Soprunov, Decidable expansions of structures. Vopr. Kibern. 134 (1988) 175-179 (in Russian).

[9] W. Thomas, The theory of successor with an extra predicate. Math. Ann. 237 (1978) 121132.

[10] W. Thomas, Ehrenfeucht games, the composition method, and the monadic theory of ordinal words. In Structures in Logic and Computer Science, A Selection of Essays in Honor of A. Ehrenfeucht. Lect. Notes Comput. Sci. 1261 (1997) 118-143. 